\title{
Detection of Brown Dwarfs with Astrometric Satellites
}

\author{
Hans-Heinrich Bernstein \\ Astronomisches Rechen-Institut, Mönchhofstraße 12 - 14, \\ D-69120 Heidelberg, Germany
}

\begin{abstract}
The first astrometric procedure for the detection of Brown Dwarfs as a double star component was accomplished for the HIPPARCOS project, using Fourier series for the description of the orbital motion of Brown Dwarfs, in connection with classical least squares techniques for the estimation of all relevant unknowns. The disadvantage of this procedure was an insufficient number of false decisions. Modern techniques of time series analysis allows a much better mathematical treatment of the physical phenomena. With respect to the large number of candidate objects and the enhanced accuracy of the new astrometric satellites, the introduction of a new approach for the astrometric detection of Brown Dwarfs is suggested.
\end{abstract}

\section{Brown Dwarf's Astrometric Signature}

Brown Dwarfs can be detected as part of an astrometric double star system, whereat, actually, the motion of the photo centre will be observed. Due to the mutual gravitational attraction, one measures the wobble of the visible primary component while the Brown Darf remains invisible. This wobble will be taken into account during the calculation of the geometric positions of the instruments targets. In principal astrometric satellites measures the large angle between two targets as outlined in Figure 1. A sophisticated reduction process delivers spherical coordinates $\psi$ and $\zeta$ of this targets based on a fixed reference great circle (RGC), which will be treated as the astrometric observations. An adjustment process delivers the classical five astrometric parameters $\alpha, \delta$ (position), $\mu_{\alpha}, \mu_{\delta}$ (proper motion) and $\varpi$ (parallax), see Bernstein (1994).

Brown Dwarfs with periods shorter than 1.5 times the satellites observing time span can be detected by the application of time series analysing techniques to the measurements directly. Brown Drwarfs with longer periods could be seen in a proper motion analysis as described by Wielen et al. (1998). In the latter case, one plots the psitions of different astrometric parameter determinations versus the time, which results in a sine curve as showed in Figure 2. The proper motions gives the different gradients of the tangents on this sine curves at the given time instants. An analysis of the different values of these gradients allows the orbit determination of an unseen companion. 


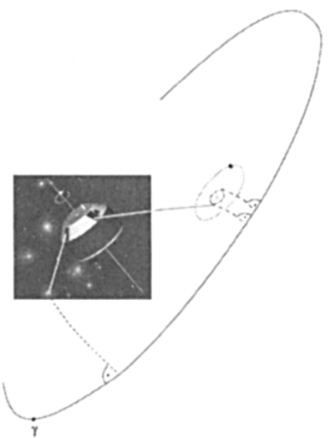

Figure 1

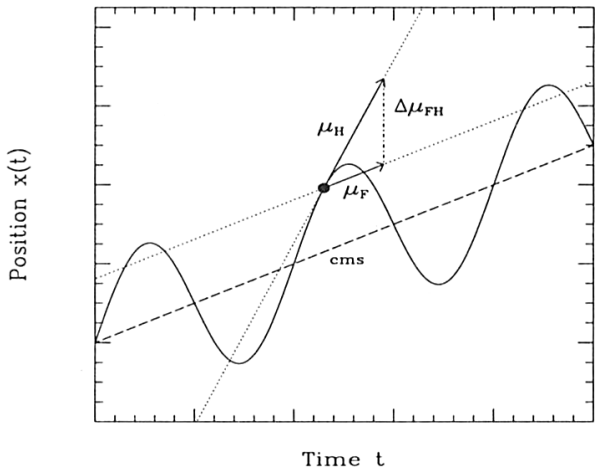

Figure 2

On the basis of Keplers third law, the capability of astrometric satellites can be estimated. The results are plotted in Figures $3 \& 4$. Figure 3 is based on HIPPARCOS data and represents the current status of the astrometric low mass detection capacity, while Figure 4 shows the attainabel situation with GAIA in the future, see Bernstein (1997) and Bernstein \& Bastian (1995).

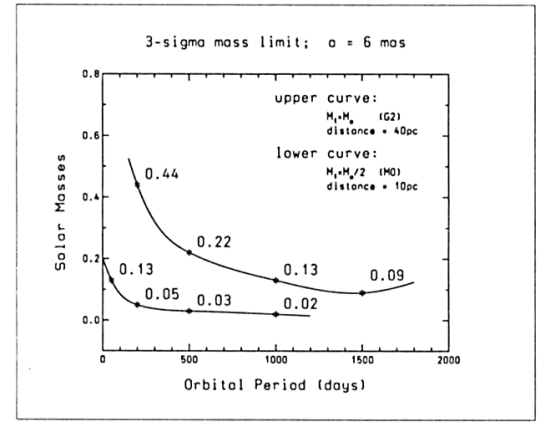

Figure 3

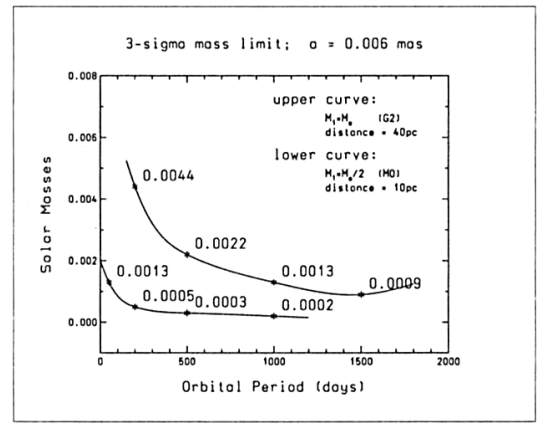

Figure 4

\section{The Orbital Searching Procedure}

The following three steps must be accomplished to get an orbit astrometrically:

1. Description of the location of the orbital plain in space by using the ThieleInnes constants A, B, F \& G:

$$
\begin{aligned}
A & =a_{p h}(+\cos \omega \cos \Omega-\sin \omega \sin \Omega \cos i) \\
B & =a_{p h}(+\cos \omega \sin \Omega+\sin \omega \cos \Omega \cos i) \\
F & =a_{p h}(-\sin \omega \cos \Omega-\cos \omega \sin \Omega \cos i) \\
G & =a_{p h}(-\sin \omega \sin \Omega+\cos \omega \cos \Omega \cos i) \\
a_{p h} & =a\left(\frac{M_{S}}{M_{P}+M_{S}}-\frac{1}{1+10^{0.4 \Delta m}}\right)
\end{aligned}
$$


$\Omega, i, \omega, a$ are the well known classical parameters for the orbital plains location in space, $M_{P}, M_{S}$ and $\Delta m$ are the masses of the primary and the secondary components and the corresponding magnitude difference.

2. Expanding the elliptical coordinates $x(t), y(t)$ in the orbital plain by Fourier series with the mean anomaly $M(t)$ as the argument and Bessel functions $J_{s}, J_{s}^{\prime}$ of the excentricity $e$ as the amplitudes:

$$
\begin{aligned}
x(t) & =-\frac{3}{2} e+2 \cdot \sum_{s=1}^{\infty} \frac{1}{s} J_{s}^{\prime}(s \cdot e) \cdot \cos (s \cdot M(t)) \\
y(t) & =\sqrt{1-e^{2}} \cdot \frac{2}{3} \cdot \sum_{s=1}^{\infty} \frac{1}{s} J_{s}(s \cdot e) \cdot \sin (s \cdot M(t)) \\
M(t) & =\tilde{M}(t)-\tilde{M}_{\circ}=\frac{2 \pi}{P}\left(t-t_{\circ}\right)
\end{aligned}
$$

Here $P$ is the period and $t_{\circ}$ is the periastron passage time of the orbit.

3. varying the $P$ or the corresponding frequency within reasonable limits and looking for the best solution: Equations 1 to 8 enters in the calculation of the geometric positions $\alpha(t)$ and $\delta(t)$ in the equations 9 to 12 :

$$
\begin{aligned}
\Delta \alpha(t) & =(B \cdot x(t)+G \cdot y(t)) / \cos \delta_{\circ} \\
\Delta \delta(t) & =(A \cdot x(t)+F \cdot y(t)) \\
\alpha(t) & =\alpha+\mu \alpha \cdot \Delta t+\varpi_{\alpha}+\Delta \alpha(t) \\
\delta(t) & =\delta+\mu \delta \cdot \Delta t+\varpi_{\delta}+\Delta \delta(t)
\end{aligned}
$$

$\varpi_{\alpha}$ and $\varpi_{\delta}$ are the corresponding parallaxe effects.

Using the transformation matrix $\mathbf{T}$ for connecting $\psi$, the observations with the location of the RGC (fixed by the corresponding coordinates $\alpha_{\wp}$ and $\delta_{\wp}$ of the RGC's pole), we get in a simple version, see Bernstein(1994):

$$
\begin{aligned}
& \psi=\arctan \left[\frac{T_{2,1} \cos \delta(t) \cos \alpha(t)+T_{2,2} \cos \delta(t) \sin \alpha(t)+T_{2,3} \sin \delta(t)}{T_{1,1} \cos \delta(t) \cos \alpha(t)+T_{1,2} \cos \delta(t) \sin \alpha(t)+T_{1,3} \sin \delta(t)}\right] \\
& \mathbf{T}=\left(\begin{array}{ccc}
\cos \alpha_{\aleph} & \sin \alpha_{\aleph} & 0 \\
-\sin \delta_{\aleph} \sin \alpha_{\aleph} & \sin \delta_{\aleph} \cos \alpha_{\aleph} & \cos \delta_{\aleph} \\
\cos \delta_{\aleph} \sin \alpha_{\aleph} & -\cos \delta_{\aleph} \cos \alpha_{\aleph} & \sin \delta_{\aleph}
\end{array}\right) \\
& \alpha_{\aleph}=\alpha_{\wp}+\pi / 2 \quad \delta_{\aleph}=\pi / 2-\delta_{\wp}
\end{aligned}
$$

With a certain set of measurments $\psi$ we estimate the five astrometric parameters within a classical least squares adjustment. The criteria for the best solution as a function of $P$ are the min. $\chi^{2}$, or here the rms of unit weight, the max. of the signal to noise ratio of the semi major axis $a$, Figure 5, the min. of the determinant of the covariance matrix of the astrometric parameters, Figure $6^{1}$ and in addition the min. of the trace and the Euclidian norm of the same matrix.

\footnotetext{
${ }^{1}$ Calculated with HIPPARCOS measurements for HIPP star No. 73184
} 


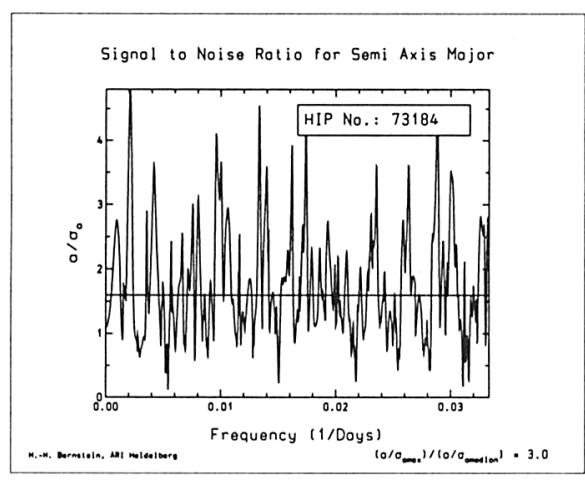

Figure 5

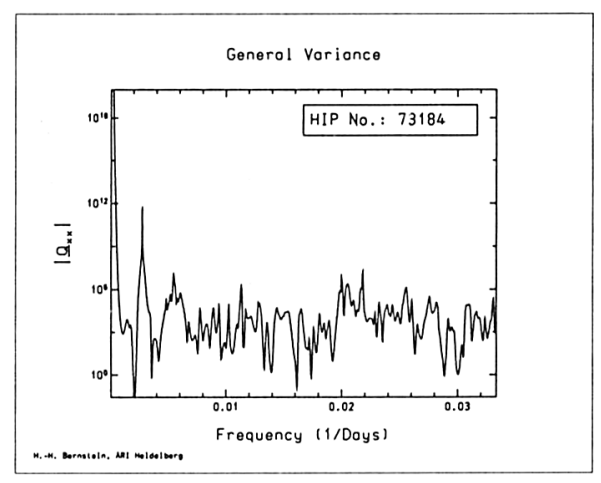

Figure 6

\section{Advantages, Problems and Solutions}

The most important advantage is the linear independency of the quantities in question with the mean anomaly in the equations 1 to 12 . No a priori values are needed, so no knowledge of the orbit is necessary. However, there is an ambiguity concerning the astrometric signature, the same curves like Figures 5 or 6 could leed to a Brown Dwarf as well as to a classical double star, when $\Delta m$ and the mass ratio is unknown in equ. 5 . In the case of HIPPARCOS one gets an estimate of $\Delta m$ from the light curve delivered from the grid of the instrument.

Such a method using time series analysing procedures could run in all problems of those procedures, e.g. the leakage effect. This leeds to unwanted falls alarm detections.

A first idea of a solution is to change the weighting system during the estimation procedure and investigate the stability of the solution. Another solution could be a two dimensional searching in $P$ and $t_{\mathrm{o}}$.

Due to the enhanced accuracy of GAIA, all orbital parameters must be treated as time dependent values and one has to investigate the influence of this fact to the period searching process and the hole orbit reconstruction.

\section{References}

Bernstein, H.-H. 1994, A\&A, 283, 293-300

Bernstein, H.-H., Bastian, U. 1995, in ESA SP-379, 55-59

Bernstein, H.-H. 1997, in ESA SP-402, 705-708

Wielen, R., Dettbarn, C., Jahreiß, H., Lenhardt, H., Schwan H. 1998, A\&A, $346,675-685$ 